\title{
Detection and molecular analysis of Campylobacter ureolyticus in domestic animals
}

\author{
Monika Koziel ${ }^{1}$, Gerard D Corcoran ${ }^{2}$, Roy D Sleator ${ }^{1 *}$ and Brigid Lucey ${ }^{1}$
}

\begin{abstract}
Previous studies showed the presence of Campylobacter ureolyticus in a large proportion of diarrhoeal samples from patients in Ireland. This emerging gastrointestinal pathogen was the second most common Campylobacter species detected in patients presenting with gastroenteritis, surpassed only by $C$. jejuni. However, the source of $C$. ureolyticus infections in humans remains unknown. The aim of this study was to investigate the presence of $C$. ureolyticus in a range of domestic animals. Over a period of 6 months, 164 samples collected from various domestic animals were tested using molecular method based on detection of the $C$. ureolyticus specific $h s p 60$ gene. These included canine faeces $(n=44)$, feline faeces $(n=31)$ and porcine faeces $(n=89)$. C. ureolyticus was detected in $32 \%(10 / 31)$ of feline faeces, 9\% (4/44) of canine faeces and 18\% (16/89) of porcine faeces. Random Amplified Polymorphic DNA (RAPD) analysis of $C$. ureolyticus isolates showed that an isolate from a cat is genetically similar to a strain isolated from a patient presenting with gastroenteritis.

This study reports the first detection and isolation of this organism in domestic animals in Ireland, with a potential source for human infection. Together with the previously reported detection of $C$. ureolyticus in bovine samples, it is likely that this emerging pathogen has a zoonotic potential.
\end{abstract}

Keywords: Campylobacter ureolyticus, Emerging pathogen, Reservoirs, Domestic animals, Zoonosis

\section{Background}

Infections with Campylobacter spp. account for a significant proportion of the reported cases of bacterial gastroenteritis worldwide [1].

Understanding the spread of campylobacter and its transmission from environmental sources to humans is essential for disease control and prevention. Campylobacter spp. have been detected in a variety of sources, such as wild birds and rivers [2,3]. However, campylobacteriosis is mostly a disease of zoonotic origin - inappropriate handling and consumption of undercooked, contaminated meat and other food products of animal origin, such as unpasteurised milk, are the most important risk factors for Campylobacter spp. infection [4]. While chickens and cattle are the most common animal reservoirs of Campylobacter spp, particularly C. jejuni and C. coli, other animals, such as pigs, sheep and dogs have also been linked to human infections [5-7].

\footnotetext{
* Correspondence: roy.sleator@cit.ie

'Department of Biological Sciences, Cork Institute of Technology, Bishopstown, Cork, Ireland

Full list of author information is available at the end of the article
}

Recently, molecular-based studies of the prevalence of various Campylobacter spp. in patients presenting with gastroenteritis in Southern Ireland, have identified Campylobacter ureolyticus as the second most common Campylobacter species, after C. jejuni [8]. Other studies have detected $C$. ureolyticus in patients suffering from diarrhoea $[9,10]$, Crohn's disease $[11,12]$ and ulcerative colitis [13].

However, despite being detected in a large proportion of patients, the possible sources of human infections still remain unclear.

In this study we investigate the presence of $C$. ureolyticus in samples obtained from animals which were previously shown to be positive for Campylobacter species such as C. jejuni and C. coli, in order to elucidate possible routes of transmission of this emerging pathogen to humans.

\section{Results}

From November 2012 to March 2013, 164 faecal samples were collected from domestic animals (dogs, cats and pigs). These were tested for $C$. ureolyticus with species- 
specific polymerase chain reaction (PCR) and the positive results were cultured. The results are presented in Table 1.

Of 89 porcine samples tested, 16 (18\%) tested positive for the presence of Campylobacter ureolyticus (Table 1). In Farm 1, the majority of the $C$. ureolyticus-positive piglets were aged $\geq 2$ months and a large proportion of piglets 6-9 days old was also positive for this organism. None of the 16 piglets aged 4-6 weeks tested positive for $C$. ureolyticus. Since no piglets younger than 2 months old were available in Farm 2 at the time of testing, no comparable data are available from this farm.

C. ureolyticus was also detected in pets in this study. A total of $32 \%$ of cat faecal samples and $9 \%$ of dog faecal samples were positive for this organism. These samples were collected from both local animals and animals in the care of two Cork veterinary hospitals.

All PCR-positive samples were inoculated on NAV medium. Among all 30 animal samples cultured, one strain of $C$. ureolyticus was isolated from cat faeces. To investigate the genetic similarity of this strain (CIT 012) to isolates obtained from patients' faecal samples and culture collection strains, RAPD analysis with $(\mathrm{GTG})_{5}$ primer was performed (Figure 1).

Gel profiles were compared and clustered on the basis of pattern similarity, using Phoretix 1D Pro software, and the obtained dendrogram is presented in Figure 2. Feline isolate CIT 012 clustered closely with the CIT 009 isolate obtained from an elderly female suffering from gastroenteritis.

Antibiotic susceptibility testing (AST) with disc diffusion method showed that strain CIT 009 and CIT 012 are resistant to ciprofloxacin, nalidixic acid and trimethoprim.

\section{Discussion}

The emerging role of Campylobacter ureolyticus as a potential cause of human gastrointestinal illness has been of interest to various research groups worldwide. However, reported isolations of $C$. ureolyticus from animals are very limited and mostly associated with endometrial infections in mares [14,15], potentially linked to infertility in these animals.

A previous study, performed by our research group, reported the presence of C. ureolyticus in a total of $13 \%$ of unpasteurised milk samples, collected across two different farms in Co. Cork, Ireland [16]. However, an

Table 1 Summary of PCR-positive results obtained throughout the study

\begin{tabular}{ccc}
\hline Sample source & $\begin{array}{c}\text { PCR positive } \\
\text { samples (\%) }\end{array}$ & $\begin{array}{c}\text { Culture positive } \\
\text { samples (\%) }\end{array}$ \\
\hline Porcine & $16(18 \%)$ & $0 / 16(0.0)$ \\
Canine & $4(9 \%)$ & $0 / 44(0.0)$ \\
Feline & $10(32 \%)$ & $1 / 31(3.2)$ \\
\hline
\end{tabular}

investigation into a zoonotic potential of this emerging pathogen and its association with domestic animals, which are common transmission vectors for other Campylobacter species such as C. jejuni, has not yet been investigated. This study was undertaken to investigate the presence of $C$. ureolyticus in domestic and companion animals using molecular and traditional approaches.

Among 89 porcine faecal swabs collected, 18\% $(\mathrm{n}=16)$ tested positive for C. ureolyticus (Table 1). A similar proportion of C.ureolyticus-positive animals was observed across the two farm sites tested with 19\% of pigs in Farm 1 and 17\% of pigs in Farm 2 positive for this organism (Table 2).

The overall positive sample rate was similar for the two farms tested, despite the fact they were separated geographically. Interestingly, a similar study by Soultos et al. [17] investigating the prevalence of Campylobacter coli in piglets aged 3-66 days and their sows reported that a majority of piglets in their first days of life were colonised with Campylobacter coli genotypes resembling those isolated from the sow. These were often displaced by other genotypes in 3 month old piglets. Many of the piglets and sows carried multiple genotypes of C. coli [17]. Since no isolates were recovered from piglets in this study, no investigation into the genetic diversity of $C$. ureolyticus strains in piglets at different age was possible.

As no other study has investigated the prevalence of C. ureolyticus in porcine samples, no direct comparison of the levels found with these reported in this study is possible. However, other studies report significant rates of fastidious Campylobacter species, such as C. concisus (9\%) or C. helveticus (6\%) in porcine caecal content tested [18]. The rates reported in this study for C. ureolyticus are comparable with those reported for other fastidious species [18]. The high prevalence of C. ureolyticus in the porcine gastrointestinal tract, highlights the potential for zoonotic transmission, especially if abattoir hygiene was not maintained at acceptable levels. This could be of particular importance for retail products, such as cured or smoked ham and bacon, which do not undergo extensive heat treatment, which would otherwise significantly decrease the number of viable bacteria in foodstuffs. However further studies are needed to determine whether consumption of such foods increases the risk for C. ureolyticus infection in humans.

Campylobacter ureolyticus was also detected in faecal samples collected from cats and dogs. These included samples collected from companion household animals as well as animals in veterinary care. A total of $9 \%$ of canine and $32 \%$ of feline samples tested positive for the presence of $C$. ureolyticus. Metagenomic studies carried out on cats, report percentages of Campylobacter spp. as high as 10\% [19]. Moreover, Campylobacter spp. of potential clinical relevance, other than $C$. jejuni and $C$. coli, such as 


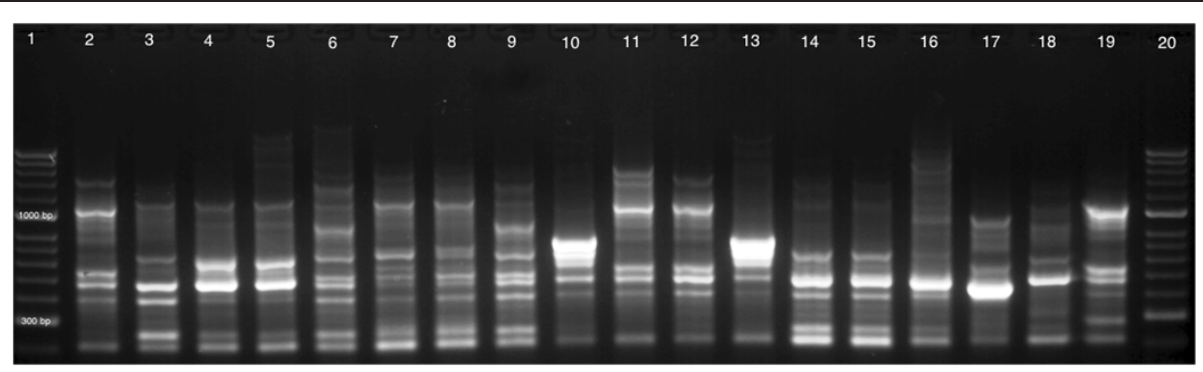

Figure 1 1.5\% agarose gel of RAPD profiles of $C$. ureolyticus isolates generated with (GTG) ${ }_{5}$ primer. Lane $1: 100$ bp molecular weight marker; Lane 2: CIT 001; Lane 3: CIT 002; Lane 4: CIT 003; Lane 5: CIT 004; Lane 6: CIT 005; Lane 7: CIT 006; Lane 8: CIT 007; Lane 9: CIT 008; Lane 10: CIT 009; Lane 11: CIT 010; Lane 12: CIT 011; Lane 13: CIT 012; Lane 14: CIT 013; Lane 15: CIT 014; Lane 16: DSMZ 20703'; Lane 17: CCUG 9510; Lane 18: CCUG 58468; Lane 19: CCUG 59897; Lane 20: 100 bp molecular weight marker.

C. upsaliensis, have been shown to be present in dog faeces [6]. PCR detection rates of Campylobacter spp. from dog faeces are highly variable (58-97\%) depending on the study, geographical location and methodology used [20].

Various factors appear to influence the level of Campylobacter spp. faecal shedding in cats and dogs. These include the age of the animal, with puppies $<6$ months and cats $<36$ months more frequently positive for Campylobacter spp. [21-23]; housing conditions, with dogs housed in kennels or shelters and outdoor cats with no access to litter trays, more at risk of campylobacter infections $[23,24]$; and dogs' diet, with a higher risk of campylobacter infection for animals fed a homemade food diet [25].

It has been shown that strains isolated from patients with Campylobacter jejuni infections, particularly young children, and their pets are genetically similar, confirming the possibility of zoonotic transmission of this genus [26-28].

This finding is of potential importance for understanding the possible sources of $C$. ureolyticus infections in humans. The majority of patients suffering from gastroenteritis in which $C$. ureolyticus was detected, were reported to be children $<5$ years old or adults $>70$ years old [29]. In this study, we have shown that the $C$. ureolyticus strain isolated from a cat's faeces is genetically similar to the isolate CIT 009 cultured from the diarrhoeal stool of an elderly patient (Figure 2). RAPD profiles of these two isolates were identical and clustered together with other strains isolated from the faeces of patients aged 83 (CIT 001), 81 (CIT 010) and 3 years old (CIT 011) as well as an isolate recovered from the blood of an elderly patient (78 years).

The isolation of Campylobacter ureolyticus from animal faeces is difficult, with an isolation rate of 3.2\% from PCRpositive cat samples and zero from pig and dog samples. The difficulty in isolation can be partially attributed to a lack of selective medium for C. ureolyticus. This organism is sensitive to many antibiotics commonly used in media supplements for $C$. jejuni or $C$. coli, such as cefoperazone or cycloheximide. Moreover, the fastidious nature of the species together with an optimal growth temperature of $37^{\circ} \mathrm{C}$, rather than $42^{\circ} \mathrm{C}$, make it more difficult to isolate than, for example, C. jejuni, especially from samples, such as faeces, which have a large number of competing microorganisms.

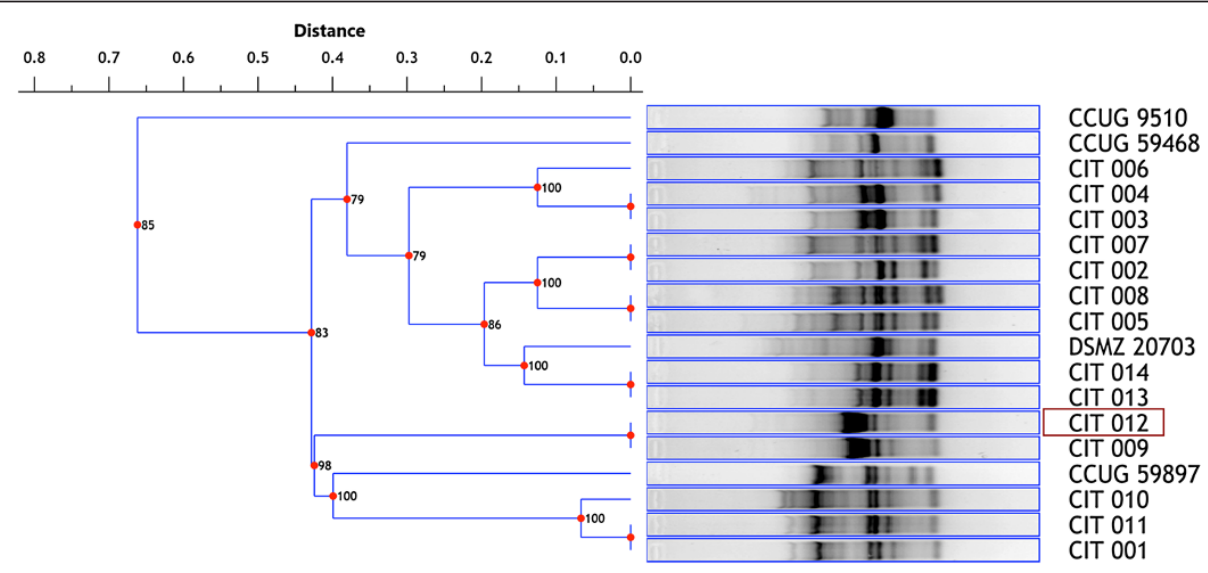

Figure 2 Dendrogram of $\boldsymbol{C}$. ureolyticus RAPD profiles. Unweighted Pair Group Method with Arithmetic Mean (UPGMA) with a dice coefficient distance tree was generated with Phoretix 1D Pro software. Highlighted isolate (CIT 012) is the feline isolate. 
Table 2 Summary of positive porcine faecal samples from the two farms sampled

\begin{tabular}{lcccc}
\hline \multicolumn{5}{c}{ Number of positive samples (\%) } \\
\hline Farm site & \multicolumn{4}{c}{ Age of piglets } \\
\cline { 2 - 5 } & $\mathbf{6 - 9}$ days & 4-6 weeks & $\mathbf{2}$ months & Total \\
\hline Farm 1 & $7 / 29(24)$ & $0 / 16(0)$ & $3 / 8(38)$ & $10 / 53(19)$ \\
Farm 2 & N/A & N/A & $6 / 36(17)$ & $6 / 36(17)$ \\
Total & $4 / 29(14)$ & $0 / 16(0)$ & $9 / 44(20)$ & $\mathbf{1 6 / 8 9 ( 1 8 )}$ \\
\hline
\end{tabular}

Culturing of Campylobacter spp. is problematic even for species such as C. jejuni or C. coli, for which use of selective temperature, selective media and well established protocols is possible. It has been previously shown that among diarrhoeal samples from patients, as much as $50 \%$ were not cultured, despite being determined to be Campylobacter jejuni and C. coli [30,31]. Therefore, the prevalence of fastidious Campylobacter species is even more likely to be underestimated when traditional culture methods, targeting mostly thermophilic species, are employed.

The difficulty in culturing campylobacters in general, may also be a attributed to the presence of organisms in a viable but nonculturable state. It has been shown that campylobacter cells which enter this state, cannot be cultured on artificial media, despite remaining virulent and capable of invading intestinal cells [32].

Molecular based detection offers an alternative, allowing us to circumvent the problems facing traditional culture based approaches. Therefore, despite a failure to grow pathogenic Campylobacter spp., their detection with molecular methods is still likely to be of clinical significance.

\section{Conclusion}

The effect of $C$. ureolyticus infections on animal health has, to date, not been investigated in any great detail. All stools samples collected throughout this study, were nondiarrhoeal, therefore no comparison of $C$. ureolyticus rates in healthy and sick animals was possible.

Nevertheless, this is the first study reporting the presence of C. ureolyticus in samples collected from domestic animals. Moreover, comparison of genetic profiles of isolates from animal and human origins shows the potential involvement of pets in C. ureolyticus infection, highlighting its potential for zoonotic transmission.

\section{Methods}

\section{Sample collection}

Faecal samples from asymptomatic pigs $(n=89)$, dogs $(\mathrm{n}=44)$ and cats $(\mathrm{n}=31)$ were collected between November 2012 and March 2013.

Porcine samples were obtained from piglets age 6-9 days old $(n=29)$, 4 weeks old $(n=16)$ and 2 months old $(\mathrm{n}=44)$ from two separate pig farms in Southern Ireland, located approximately $20 \mathrm{~km}$ apart. Piglets were swabbed using sterile swabs (Deltalab) and the faecal samples were placed in $15 \mathrm{ml}$ conical tubes (Corning) filled to the top with Bolton broth (Oxoid) with NAV supplement [33] which consisted of $\left(\mathrm{l}^{-1}\right): 2 \mathrm{~g}$ sodium formate (Fluka), $3 \mathrm{~g}$ sodium fumarate (Fluka), $10 \mathrm{mg}$ of amphotericin B, $10 \mathrm{mg}$ nalidixic acid and $20 \mathrm{mg}$ of vancomycin (all supplied by Sigma Aldrich).

Canine and feline samples were collected from local domestic animals and animals cared for in two separate veterinary hospitals in Co. Cork. Fresh faecal matter was placed in $25 \mathrm{ml}$ centrifuge tube (Sarstedt) filled with Bolton Broth with NAV supplement.

\section{Sample preparation}

All collected samples were immediately closed tightly (to create anaerobic conditions) and transported to the laboratory for incubation.

Samples were incubated aerobically at $37^{\circ} \mathrm{C}$ for a minimum of 7 days. Following incubation, samples were tested for the presence of $C$. ureolyticus using PCR. Remaining enriched broths were kept at $37^{\circ} \mathrm{C}$ for culture of any positive results following molecular analysis.

\section{Molecular detection}

Following incubation, $2 \mathrm{ml}$ of enriched sample was taken for molecular analysis. Samples were centrifuged at $17,000 \times \mathrm{g}$ for 1 minute and bacterial DNA from the pellet was extracted with QIAamp DNA Stool Mini Kit (Qiagen). The presence of C. ureolyticus was investigated as previously described [16]. In brief, C. ureolyticusspecific primers targeting the $h s p 60$ gene of this organism [34] were used for analysis of the samples. Potential PCR inhibition by the sample was monitored by including an internal amplification control (IAC) in every PCR reaction.

All PCR reactions consisted of $1 \mathrm{U}$ of HotStarTaq Plus DNA Polymerase (Qiagen), $1 \mu \mathrm{M}$ of each primer (Eurofins, MWG Operon), $0.2 \mu \mathrm{M}$ of each dNTPs (Sigma Aldrich) and $100 \mathrm{ng}$ of DNA template. The C. ureolyticus DSMZ 20703 type strain was used as a positive control for both extraction and PCR amplification. PCR conditions were as suggested by the Taq manufacturer and the annealing temperature was set at $58^{\circ} \mathrm{C}$ for 1 minute. Amplicons were visualised on $1.5 \%$ agarose gels and the results were interpreted on the basis of presence/absence of a band of $429 \mathrm{bp}$ for C. ureolyticus positive samples and IAC amplification (196 bp) for C. ureolyticus negative samples. Positive amplicons were purified using QIAquick PCR Purification Kit (Qiagen) and PCR positive products were sequenced (GATC Biotech) using the $h s p 60$ primers to ensure the validity of the positive results. 


\section{Culture methods}

Samples were cultured immediately, once presumptive positive results from the PCR were obtained, to attempt recovery of $C$. ureolyticus. Enriched samples were inoculated onto NAV agar [33], consisting of $\left(1^{-1}\right) 46 \mathrm{~g}$ of Anaerobe Basal Agar (Oxoid), 10 g Agar (Sigma Aldrich) and NAV supplement described previously. Each sample was inoculated in triplicate and incubated anaerobically using AnaeroGen 2.5 L gas packs (Oxoid) at $37^{\circ} \mathrm{C}$ for up to 14 days. Plates were checked during that period at regular intervals (3-4 days) for presumptive C. ureolyticus colonies: flat, translucent, spreading colonies. Colony PCR with C. ureolyticus - hsp60 primers was performed on putative colonies. All PCR positive isolates were sent for $16 \mathrm{~S} r R N A$ sequencing using $\mathrm{fD} 1$ and $\mathrm{rP} 1$ primers [35] to confirm their identity.

\section{RAPD analysis and comparison with human isolates}

Campylobacter ureolyticus strains isolated from animals were compared with human isolates obtained from patients presenting with gastroenteritis, healthy controls and strains available from culture collections. Details of the isolates are shown in Table 3.

To obtain RAPD profiles, PCR reactions were carried out with $(\mathrm{GTG})_{5}$ primer [36] at a final concentration of $1 \mu \mathrm{M}, 1 \mathrm{U}$ GoTaq DNA Polymerase (Promega), $0.2 \mu \mathrm{M}$ of each dNTPs and $100 \mathrm{ng}$ of template DNA. Thermal cycling conditions were as follows: 1 initial denaturation cycle at $94^{\circ} \mathrm{C}$ for $5 \mathrm{~min}$, followed by 34 cycles of denaturation at $94^{\circ} \mathrm{C}$ for $2 \mathrm{~min}$, annealing as described later and extension at $72^{\circ} \mathrm{C}$ for $2 \mathrm{~min}$ followed by the final extension step at $72^{\circ} \mathrm{C}$ for $5 \mathrm{~min}$. To improve reproducibility, the first 4 cycles were performed at lower stringency, at annealing temperature of $36^{\circ} \mathrm{C}$ for $2 \mathrm{~min}$, followed by 30 cycles at $50^{\circ} \mathrm{C}$ for $1 \mathrm{~min}$. PCR amplicons were separated on $1.5 \%$ agarose electrophoresed at $70 \mathrm{~V}$ for $90 \mathrm{~min}$. The gels were stained with ethidium bromide and visualised with UV transluminator (UVP, Cambridge, UK). Gels were analysed using Phoretix 1D Pro software (TotalLab) and similarities of RAPD fingerprints of all isolates were illustrated as clusters based on the banding patterns.

Antibiotic susceptibility of isolated CIT 009 and CIT 012 was determined using the disc diffusion method with the following discs tested: ciprofloxacin $(30 \mu \mathrm{g})$, nalidixic acid $(30 \mu \mathrm{g})$ and trimethoprim $(5 \mu \mathrm{g})$. Both strains were susceptible to tetracycline $(20 \mu \mathrm{g})$, erythromycin $(15 \mu \mathrm{g})$, gentamicin $(10 \mu \mathrm{g})$ and cefoperazone $(30 \mu \mathrm{g})$ (all discs were supplied by Oxoid). Bacterial suspension were adjusted to 0.5 McFarland in PBS and spread onto Mueller Hinton agar (Sigma) supplemented with 5\% defibrinated horse blood (TCS Biosciences). Plates were incubated anaerobically for 48 hours; in case of insufficient growth, plates were re-incubated for further 24 hours. Zone diameters were interpreted accordingly to recommendations of the European Committee on Antimicrobial Susceptibility

Table 3 Summary of the strains used in this study

\begin{tabular}{|c|c|c|c|}
\hline Strain & Gender (Age in years) & Sample source & Medical history \\
\hline CIT 001 & Male (83) & Human faeces & Long term hospital stay \\
\hline CIT 002 & Female (84) & Human faeces & End stage chronic renal disease \\
\hline CIT 003 & Male (65) & Human faeces & Nursing home resident. Recurring diarrhoea \\
\hline CIT 004 & Female (3) & Human faeces & Admitted to the hospital with diarrhoea and vomiting. Cryptosporidium oocyst detected. \\
\hline CIT 005 & Female (3) & Human faeces & Positive for cryptosporidium oocysts \\
\hline CIT 006 & Female (71) & Human faeces & Long term hospital stay \\
\hline CIT 007 & Female (84) & Human faeces & End stage chronic renal disease. Recurring diarrhoea \\
\hline CIT 008 & Female (55) & Human faeces & Hepatic cirrhosis/diabetes \\
\hline CIT 009 & Female (83) & Human faeces & Nursing home resident \\
\hline CIT 010 & Female (81) & Human faeces & Nursing home resident \\
\hline CIT 011 & Female (3) & Human faeces & $\mathrm{N} / \mathrm{A}$ \\
\hline CIT 012 & Female (2) & Cat faeces & asymptomatic, healthy (This study) \\
\hline CIT 013 & Female (26) & Human faeces & asymptomatic, healthy \\
\hline CIT 014 & Male (33) & Human faeces & asymptomatic, healthy \\
\hline DSMZ $20703^{T_{*}}$ & Female & Amniotic fluid & $\mathrm{N} / \mathrm{A}$ \\
\hline CCUG 9510* & Male (22) & Penis wound & $\mathrm{N} / \mathrm{A}$ \\
\hline CCUG 59468* & Female & Vagina & $\mathrm{N} / \mathrm{A}$ \\
\hline CCUG 59897* & Unknown (78) & Human blood & N/A \\
\hline
\end{tabular}

*Strains were obtained from Culture Collection, University of Göteborg, Sweden (CCUG) and Leibniz Institute DSMZ-German Collection of Microorganisms and Cell Cultures, Braunschweig, Germany. 
Testing [37], the British Society for Antimicrobial Chemotherapy [38] and breakpoints used for Campylobacter spp. in other studies [39]. In the absence of such information, breakpoints were decided by authors as susceptible $\geq 10 \mathrm{~mm}$ and resistant $\leq 10 \mathrm{~mm}$.

\section{Abbreviations}

PCR: Polymerase Chain Reaction; RAPD: Random Amplified Polymorphic DNA; MLST: Multilocus Sequence Typing; NAV: Nalidixic acid, amphotericin, vancomycin; UPGMA: Unweighted Pair Group Method with Arithmetic Mean; N/A: Not applicable.

\section{Competing interests}

The authors declare that they have no competing interests.

\section{Authors' contributions}

MK designed the study, carried out the experimental work, performed the analysis, and drafted the manuscript. RDS and BL designed and coordinated the study, and edited the manuscript. GDC edited the manuscript. All authors read and approved the final manuscript.

\section{Acknowledgements}

The authors would like to acknowledge the assistance of the veterinary surgeons Clare Meade and Pat O'Doherty and the staff at the Cat Hospital and Gilabbey Veterinary Hospital, Cork in animal sample collection, in addition to the staff at the Microbiology Department at Cork University Hospital for help in isolating the strains used in this study. We would also like to thank Dr. P. J. Collins, Sebastien Lambert and Alan Lucid for assistance with the specimen collection. M.K. is a recipient of a scholarship provided by Irish Research Council (RS/2011/264). R.D.S. is an ESCMID Research Fellow and coordinator of the EU FP7 ClouDx-i project.

\section{Author details}

'Department of Biological Sciences, Cork Institute of Technology, Bishopstown, Cork, Ireland. 'Department of Diagnostic Microbiology, Cork University Hospital, Wilton, Cork, Ireland.

\section{Received: 10 March 2014 Accepted: 10 April 2014} Published: 16 April 2014

\section{References}

1. Allos BM: Campylobacter jejuni infections: update on emerging issues and trends. Clin Infect Dis 2001, 32:1201-1206.

2. Waldenstrom J, Axelsson-Olsson D, Olsen B, Hasselquist D, Griekspoor P, Jansson L, Teneberg S, Svensson L, Ellstrom P: Campylobacter jejuni colonization in wild birds: results from an infection experiment. PLoS One 2010, 5:e9082

3. Daczkowska-Kozon E, Brzostek-Nowakowska J: Campylobacter spp. in waters of three main western Pomerania water bodies. Int I Hyg Environ Health 2001, 203:435-443.

4. Danis K, Di Renzi M, O'Neill W, Smyth B, McKeown P, Foley B, Tohani V, Devine M: Risk factors for sporadic Campylobacter infection: an all-Ireland case-control study. Euro Surveill 2009, 14(7):19123.

5. Wilson DJ, Gabriel E, Leatherbarrow AJ, Cheesbrough J, Gee S, Bolton E, Fox A, Fearnhead P, Hart CA, Diggle PJ: Tracing the source of campylobacteriosis. PLoS Genet 2008, 4:e1000203.

6. Mughini Gras L, Smid JH, Wagenaar JA, de Boer AG, Havelaar AH, Friesema $\mathbb{H}$, French NP, Busani L, van Pelt W: Risk factors for campylobacteriosis of chicken, ruminant, and environmental origin: a combined case-control and source attribution analysis. PLoS One 2012, 7:e42599.

7. Kittl S, Heckel G, Korczak BM, Kuhnert P: Source attribution of human Campylobacter isolates by MLST and fla-typing and association of genotypes with quinolone resistance. PLOS One 2013, 8:e81796.

8. Bullman S, O'Leary J, Corcoran D, Sleator RD, Lucey B: Molecular-based detection of non-culturable and emerging campylobacteria in patients presenting with gastroenteritis. Epidemiol Infect 2012, 140:684-688.

9. Inglis $G D$, Boras VF, Houde A: Enteric campylobacteria and RNA viruses associated with healthy and diarrheic humans in the Chinook health region of southwestern Alberta, Canada. J Clin Microbiol 2011, 49:209-219.
10. Collado L, Gutierrez M, Gonzalez M, Fernandez H: Assessment of the prevalence and diversity of emergent campylobacteria in human stool samples using a combination of traditional and molecular methods. Diagn Microbiol Infect Dis 2013, 75:434-436.

11. Zhang L, Man SM, Day AS, Leach ST, Lemberg DA, Dutt S, Stormon M, Otley A, O'Loughlin EV, Magoffin A, Ng PHY, Mitchell H: Detection and isolation of Campylobacter species other than C. jejuni from children with Crohn's disease. J Clin Microbiol 2009, 47:453-455.

12. Man SM, Zhang L, Day AS, Leach ST, Lemberg DA, Mitchell H: Campylobacter concisus and other Campylobacter species in children with newly diagnosed Crohn's disease. Inflamm Bowel Dis 2010, 16:1008-1016.

13. Mukhopadhya I, Thomson JM, Hansen R, Berry SH, El-Omar EM, Hold GL: Detection of Campylobacter concisus and other Campylobacter species in colonic biopsies from adults with ulcerative colitis. PLoS One 2011, 6:e21490.

14. Hariharan H, Richardson G, Horney B, Heaney S, Bryenton J, Moore I: Isolation of Bacteroides ureolyticus from the equine endometrium. J Vet Diagn Invest 1994, 6:127-130.

15. Fodor L, Szenci O, Peters M, Varga J, Szemeredi G, Wyszoczky F: Isolation of Bacteroides ureolyticus from vaginal discharge of mares. Zentralbl Veterinarmed B 1995, 42:415-420.

16. Koziel M, Lucey B, Bullman S, Corcoran GD, Sleator RD: Molecular-based detection of the gastrointestinal pathogen Campylobacter ureolyticus in unpasteurized milk samples from two cattle farms in Ireland. Gut Pathog 2012, 4:14.

17. Soultos N, Madden RH: A genotyping investigation of the colonization of piglets by Campylobacter coli in the first 10 weeks of life. J Appl Microbiol 2007, 102:916-920.

18. Scanlon KA, Cagney C, Walsh D, McNulty D, Carroll A, McNamara EB, McDowell DA, Duffy G: Occurrence and characteristics of fastidious Campylobacteraceae species in porcine samples. Int J Food Microbiol 2013, 163:6-13.

19. Ritchie $L E$, Steiner JM, Suchodolski JS: Assessment of microbial diversity along the feline intestinal tract using $16 \mathrm{~S}$ rRNA gene analysis. FEMS Microbiol Ecol 2008, 66:590-598.

20. Marks SL, Rankin SC, Byrne BA, Weese JS: Enteropathogenic bacteria in dogs and cats: diagnosis, epidemiology, treatment, and control. J Vet Intern Med 2011, 25:1195-1208.

21. Stafford RJ, Schluter P, Kirk M, Wilson A, Unicomb L, Ashbolt R, Gregory J, OzFoodNet Working Group: A multi-centre prospective case-control study of campylobacter infection in persons aged 5 years and older in Australia. Epidemiol Infect 2007, 135:978-988.

22. Torre $\mathrm{E}$, Tello M: Factors influencing fecal shedding of Campylobacter jejuni in dogs without diarrhea. Am J Vet Res 1993, 54:260-262.

23. Wieland B, Regula G, Danuser J, Wittwer M, Burnens AP, Wassenaar TM, Stark KD: Campylobacter spp. in dogs and cats in Switzerland: risk factor analysis and molecular characterization with AFLP. J Vet Med B Infect Dis Vet Public Health 2005, 52:183-189.

24. Baker J, Barton MD, Lanser J: Campylobacter species in cats and dogs in South Australia. Aust Vet J 1999, 77:662-666.

25. Leonard EK, Pearl DL, Janecko N, Weese JS, Reid-Smith RJ, Peregrine AS, Finley RL: Factors related to Campylobacter spp. carriage in client-owned dogs visiting veterinary clinics in a region of Ontario, Canada. Epidemiol Infect 2011, 139:1531-1541.

26. Damborg P, Olsen KE, Moller Nielsen E, Guardabassi L: Occurrence of Campylobacter jejuni in pets living with human patients infected with C. jejuni. J Clin Microbiol 2004, 42:1363-1364.

27. Wolfs TF, Duim B, Geelen SP, Rigter A, Thomson-Carter F, Fleer A, Wagenaar JA: Neonatal sepsis by Campylobacter jejuni: genetically proven transmission from a household puppy. Clin Infect Dis 2001, 32:E97-E99.

28. Wardak S, Duda U, Wojsa B: [Molecular investigation of relatedness of Campylobacter coli isolated from child with campylobacteriosis and healthy, household dog]. Medycyna doswiadczalna i mikrobiologia 2009, 61:235-241.

29. Bullman S, Corcoran D, O'Leary J, O'Hare D, Lucey B, Sleator RD: Emerging dynamics of human campylobacteriosis in Southern Ireland. FEMS Immunol Med Microbiol 2011, 63:248-253.

30. Bessede E, Delcamp A, Sifre E, Buissonniere A, Megraud F: New methods for detection of campylobacters in stool samples in comparison to culture. J Clin Microbiol 2011, 49:941-944. 
31. Onori M, Coltella L, Mancinelli L, Argentieri M, Menichella D, Villani A, Grandin A, Valentini D, Raponi M, Russo C: Evaluation of a multiplex PCR assay for simultaneous detection of bacterial and viral enteropathogens in stool samples of paediatric patients. Diagn Microbiol Infect Dis 2014. S0732-8893(14)00067-4.

32. Chaisowwong W, Kusumoto A, Hashimoto M, Harada T, Maklon K, Kawamoto K: Physiological characterization of Campylobacter jejuni under cold stresses conditions: its potential for public threat. J Vet Med Sci 2012, 74:43-50.

33. O'Doherty A, Koziel M, De Barra L, Corcoran D, Bullman S, Lucey B, Sleator RD: Development of nalidixic acid amphotericin B vancomycin (NAV) medium for the isolation of Campylobacter ureolyticus from the stools of patients presenting with acute gastroenteritis. Br J Biomed Sci 2014, 71:6-12.

34. Bullman S, Corcoran D, O'Leary J, Lucey B, Byrne D, Sleator RD: Campylobacter ureolyticus: an emerging gastrointestinal pathogen? FEMS Immunol Med Microbiol 2011, 61:228-230.

35. Weisburg WG, Barns SM, Pelletier DA, Lane DJ: 16S ribosomal DNA amplification for phylogenetic study. J Bacterio/ 1991, 173:697-703.

36. Matsheka MI, Lastovica AJ, Zappe H, Elisha BG: The use of (GTG)5 oligonucleotide as an RAPD primer to type Campylobacter concisus. Lett Appl Microbiol 2006, 42:600-605.

37. Breakpoint Tables for Interpretation of MICs and Zone Diameters. [http:// www.eucast.org/fileadmin/src/media/PDFs/EUCAST_files/Breakpoint_tables/ Breakpoint_table_v_3.1.pdf]

38. BSAC Methods for Antimicrobial Susceptibility Testing. [http://bsac.org uk/wp-content/uploads/2012/02/Nersion-11.1-2012-Final-.pdf]

39. Luangtongkum T, Morishita TY, El-Tayeb AB, Ison AJ, Zhang Q: Comparison of antimicrobial susceptibility testing of Campylobacter spp. by the agar dilution and the agar disk diffusion methods. J Clin Microbiol 2007, 45:590-594.

doi:10.1186/1757-4749-6-9

Cite this article as: Koziel et al: Detection and molecular analysis of

Campylobacter ureolyticus in domestic animals. Gut Pathogens 2014 6:9.

\section{Submit your next manuscript to BioMed Central and take full advantage of:}

- Convenient online submission

- Thorough peer review

- No space constraints or color figure charges

- Immediate publication on acceptance

- Inclusion in PubMed, CAS, Scopus and Google Scholar

- Research which is freely available for redistribution 